\section{Lessons from prenatal care provider-based recruitment into the National Children's Study}

\author{
James M. Robbins, Melissa D. Bridges, \\ Elizabeth M. Childers, \\ Roseanne M. Harris, Pearl A. McElfish \\ University of Arkansas for Medical \\ Sciences - Northwest, Fayetteville, AR, \\ USA
}

\begin{abstract}
In response to recruitment difficulties experienced by the National Children's Study, alternatives to the door-to-door recruitment method were pilot tested. This report describes outcomes, successes, and challenges of recruiting women through prenatal care providers in Benton County, Arkansas, USA. Eligible women residing in 14 randomly selected geographic segments were recruited. Data were collected during pregnancy, at birth, and at 3, 6, 9, 12, 18, and 24 months postpartum. Participants were compared to non-enrolled eligible women through birth records. Of 6402 attempts to screen for address eligibility, 468 patients were potentially eligible. Of 221 eligible women approached to participate, 151 (68\%) enrolled in the 21-year study. Enrolled women were similar to non-enrolled women in age, marital status, number of prenatal care visits, and gestational age and birth weight of the newborn. Women enrolled from public clinics were more likely to be Hispanic, lower educated, younger and unmarried than those enrolled from private clinics. Sampling geographic areas from historical birth records failed to produce expected equivalent number of births across segments. Enrollment of pregnant women from prenatal care providers was successful.
\end{abstract}

\section{Introduction}

The National Children's Study (NCS) originated in response to the Children's Health Act of 2000 which mandated a longitudinal study of the effects of the environment on children's health in the United States. ${ }^{1}$ The study is intended to generate a nationally representative probability sample of children recruited as early in their lives as possible, preferably during the early stages of pregnancy. As it was originally conceptualized, the NCS would follow a sample of 100,000 children, born to women recruited from about 105 counties within the US, from before birth to age 21 years. ${ }^{2}$

Seven original vanguard centers implemented the study beginning in 2007 using a household-based sampling and door-to-door recruitment approach. The goal of the study at each site was to recruit five pregnant women per week over a 17-20 month period. Because of slower than expected enrollment, new recruitment at these centers was suspended after only 1 year of operation. In response to perceived difficulties in recruitment experienced by the seven centers, alternatives to the doorto-door recruitment method were established. In early 2011 , thirty new study locations were added to test alternate recruitment methods. Benton County, Arkansas, was one of ten NCS study locations that began testing the recruitment of women from prenatal care providers. Partly as a result of lessons learned from these experiences, birth cohort research studies may benefit from recruitment of women from prenatal care providers instead of householdbased recruitment. ${ }^{3}$ The unique NCS providerbased sampling design tested here allowed for recruitment of all willing pregnant women living in defined geographic areas of the county. This report describes the outcomes, successes, and challenges of the Benton County Study Center's recruitment efforts from the offices of private practice obstetrician-gynecologists and practices serving publically insured women.

\section{Materials and Methods}

\section{Procedure}

The provider-based recruitment pilot study was intended to proceed until 100 participants were enrolled or until a steady state, defined as three consecutive weeks of approximately equal enrollment, was reached. Women were to be recruited from the offices of prenatal care providers as early in pregnancy as possible. While instructions allowed recruitment of prepregnant women, this population was deemphasized in favor of pregnant women. For those who were found to be age and geographically eligible, an appointment was made to obtain consent and potentially administer the first pregnancy interview. Subsequent data collection occurred in the third trimester of pregnancy, at the birth of the baby, and at $3,6,9,12$, 18 and 24 months postpartum. This study protocol was approved by the Institutional Review Board (IRB) of the University of Arkansas for Medical Sciences (UAMS). Data were primarily collected face-to-face utilizing computer assisted interviewing methods. Domains of inquiry included maternal medical history, pregnancy intentions, tobacco and alcohol use, employment and social support, housing characteristics, health insurance, pets in the home, and participant verification. Interviews after the birth of
Correspondence: Correspondence: Melissa D. Bridges, University of Arkansas for Medical Sciences - Northwest, 1125 North College Ave., Fayetteville, AR 72701, USA

Tel.: +1.479.713.8679 - Fax: +1.479.713.8670

E-mail:mdbridges@uams.edu

Key words: National Children's Study; providerbased recruitment; prenatal care; geographic sampling; retention, birth-cohort.

Acknowledgements: The study was conducted as part of the National Children's Study funded by the Office of the Director of the National Institutes of Health. Support was provided by the National Institute of Child Health and Human Development: Contract Number HHSN275200 800026C. Additional support was provided by Arkansas Children's Hospital Research Institute and the Pamela Stephens Chair in Birth Defects Research at Arkansas Children's Hospital. We thank the women of Benton County who willingly volunteered for the study and the prenatal care providers who invited the study team into their busy offices. We thank study team members Sherry Lloyd, Lisa Mann, Irasema Roldan, Angie Hunton, Amy McCoy, Amy Shova, Trace Henley, Jennifer Worth, Elizabeth Burns, Natalie Tate, Mary Jean Kippenbrock, Bridget Mosley, and Ashley Block who ensured the smooth operation and success of the study, Elizabeth Bowen who conducted the geographic segmentation of Benton County, William Hogan who developed the Comprehensive Research Informatics Suite for the study, and Charlotte A. Hobbs who was the Principal Investigator for the Benton County Study Center.

Contributions: $\mathrm{MB}$ and EC performed data management and analysis; JR, MB, EC, RH, PAM wrote and edited the manuscript.

Conflict of interest: the authors declare no conflict of interest.

Received for publication: 4 June 2015 .

Revision received: 22 July 2015 .

Accepted for publication: 22 July 2015.

This work is licensed under a Creative Commons Attribution NonCommercial 3.0 License (CC BYNC 3.0).

(C)Copyright J. M. Robbins et al., 2015

Licensee PAGEPress, Italy

Pediatric Reports 2015; 7:6056

doi:10.4081/pr.2015.6056

the baby included questions about environmental exposures, infant feeding, infant sleep, well baby care, immunizations, work plans, crying patterns, parenting, financial security, and health conditions. Following procedures employed in the initial household-based sampling technique, Benton County was divided into 126 geographic areas called segments intended 
to produce approximately equal numbers of births per year. Birth records for the six-year period 2001-2006 were geocoded according to place of residence of the mother to place births into segments. Segments were formed using natural geographic boundaries demarcating neighborhoods in densely populated areas and towns or villages in rural areas. From 126 identified segments with approximately equal number of historical births over the six-year period (110-150) 14 segments were randomly selected to comprise the secondary sampling unit (SSU). Benton County includes five small cities of population between 16,000 and 60,000 . These cities comprise about $70 \%$ of the county population. The remainder of the county is rural or smaller towns. Of the 14 segments randomly chosen for inclusion, the boundaries of 9 segments were within one of the five small cities. The remaining 5 segments were rural areas. Of the 14 randomly selected segments 4 were within 1 mile of participating prenatal care provider offices, 8 were between 3 and 5 miles from participating offices, and 2 segments were more than 12 miles from the nearest participating provider office.

\section{Determining eligibility and approaching potential participants}

Research or clinic staff identified prenatal patients with eligible addresses and flagged those charts with an NCS brochure and recruitment form. Address eligibility was determined utilizing a web-based address look-up tool. Address eligible patients were approached for participation and consent at the clinic visit. All pregnant women ages 18 to 49 living within the chosen SSU were eligible for recruitment. Eligible women were given the opportunity to consent at this visit or at a later date. Research staff operated in pairs: a trained research associate data collector, and a research registered nurse. Registered nurses were key to understanding how provider offices and hospitals functioned and how the NCS could be integrated into clinic operations while disrupting patient flow as little as possible. Using a case management approach, each research staff pair remained assigned to and conducted subsequent visits with the participants they enrolled. A $\$ 25$ gift card was distributed at each visit as remuneration for participation in the study.

\section{Data capture}

The Comprehensive Research Informatics Suite (CRIS) was used for data collection and management. CRIS was developed uniquely for the Benton County NCS Study Center by the University of Arkansas for Medical Sciences Bioinformatics Department. CRIS is a comprehensive set of open source software tools for electronic management of participant data. All components of the UAMS CRIS are web-based, enabling remote data capture. All applications are integrated into a portal that allows single point of access through a Federal Information Security Management Act (FISMA) secure sign on.

\section{Analyses}

Arkansas birth records that corresponded to the timeframe during which enrolled women delivered (January 2011-March 2012) were accessed to identify all births to women residing in eligible geographic segments of the county. Characteristics of enrolled women were compared to those of eligible but not enrolled using binary logistic regression. The number of births by SSU was calculated to determine whether stratification into geographic segments using historical birth data yielded anticipated numbers of births. Characteristics of women enrolled from public clinics were compared to those of women enrolled from private clinics by chi-square.

\section{Engagement of prenatal care providers}

Providers of health care to prenatal and preconceptional women were identified through contacts with the county medical society and local provider directories. Nine prenatal care provider clinics (at 12 locations) provide the vast majority of care to pregnant women in Benton County. The Benton County NCS office employed a medical liaison (RMH) to negotiate with providers and provider groups. To engage obstetricians, gynecologists, and family medicine physicians, the medical liaison met with each prenatal care practice and hospital to recruit them as research partners, establish subcontracts and memorandums of agreement, and detail customized standard operating procedures for participant recruitment unique to each site.

Hospital engagement was also necessary to ensure data collection during the birth event. Memoranda of understanding were established and customized standard operating procedures were written for each of four birthing hospitals. All hospitals agreed to participate and deferred to the UAMS IRB. None of the hospitals requested reimbursement for their participation.

\section{Results}

All prenatal care provider offices in Benton County agreed to participate in the National Children's Study. In total, there were over 50 individual providers practicing in the nine offices. These providers include six private provider offices staffed by obstetrician-gynecologists, two Federally Qualified Community Health Centers, and the local Benton County
Health Department. All offices provided specific research office space for NCS recruitment purposes. Three of the nine provider offices requested reimbursement for staff time and space. The provider engagement process began in September of 2010 and the first provider office began recruitment in January 2011. The last provider office began recruitment in July 2011. As instructed by the Program Office, active recruitment ended in October 2012. The Study Center was allowed to enroll additional women who called in to the office to volunteer for the study through December 2012.

\section{Subject recruitment}

A summary of the procedural steps of study recruitment is presented in Figure 1. During the active recruitment period, January 2011 through October 2012, 6402 attempts were made by clinic or NCS staff to identify women who were potentially eligible for the NCS based on their home address. Of the 6402 addresses reviewed, 468 belonged to women living in one of the 14 selected segments. Of these 468 address eligible women, 342 were approached to request participation. The remaining 126 included 96 women who were not approached due to age ineligibility, miscarriage or premature birth, language barriers, or delays in availability of Spanish instruments, and 30 women who we attempted to approach but were unsuccessful, most often due to repeated missed clinic visits or logistical or timing problems in the clinic that prevented an adequate discussion of the study with potential participants. Of 342 women approached, 252 (73.7\%) agreed to further contact from NCS staff to learn more about the study. Initial refusals $(n=90)$ included women who were not interested in the study as communicated by them to the doctor, clinic receptionist, or data collector, or as indicated on recruitment forms. Of 252 contacted women, 177 agreed to an initial study eligibility screener. Fifteen women, after discussing the study, were not interested in completing the initial eligibility screener and the remaining 31 were determined to be ineligible due to age, delivery prior to contact, no plans for pregnancy, or inability to conceive. After subtracting 31 ineligible women from the 252 who gave permission to be contacted, 177 of 221 (80.1\%) agreed to complete the initial screener. Of 177 women who completed the initial eligibility screener, 151 or $85.3 \%$ consented to participation, and 26 refused to enroll. Of the enrolled women, 147 women were pregnant and 4 women were not pregnant but trying to conceive. Overall, of 221 eligible women contacted about the study, 151, or $68.3 \%$, consented to participate in the NCS.

Retention of participant mothers of enrolled children and completion of data collection events is summarized in Table 1. Over the 
nearly two and a half year period of recruitment and data collection, the Benton County study center retained over $95 \%$ of participants and captured almost all eligible data collection events. Data collection beyond 12 months is ongoing with a number of subjects remaining in the data collection window.

\section{Characteristics of enrolled subjects}

Characteristics of mothers of enrolled children are compared to non-enrolled age and segment eligible (i.e., geographic area of residence within the county) mothers in Table 2. Enrolled mothers were similar to non-enrolled mothers in age, marital status, number of pre- natal visits, and gestational age and birth weight of their newborns. When adjusted for other characteristics, enrolled women were significantly less likely to be of Asian or Pacific Islander ethnicity and more likely than nonenrolled women to have a college degree.

Table 3 compares characteristics of women

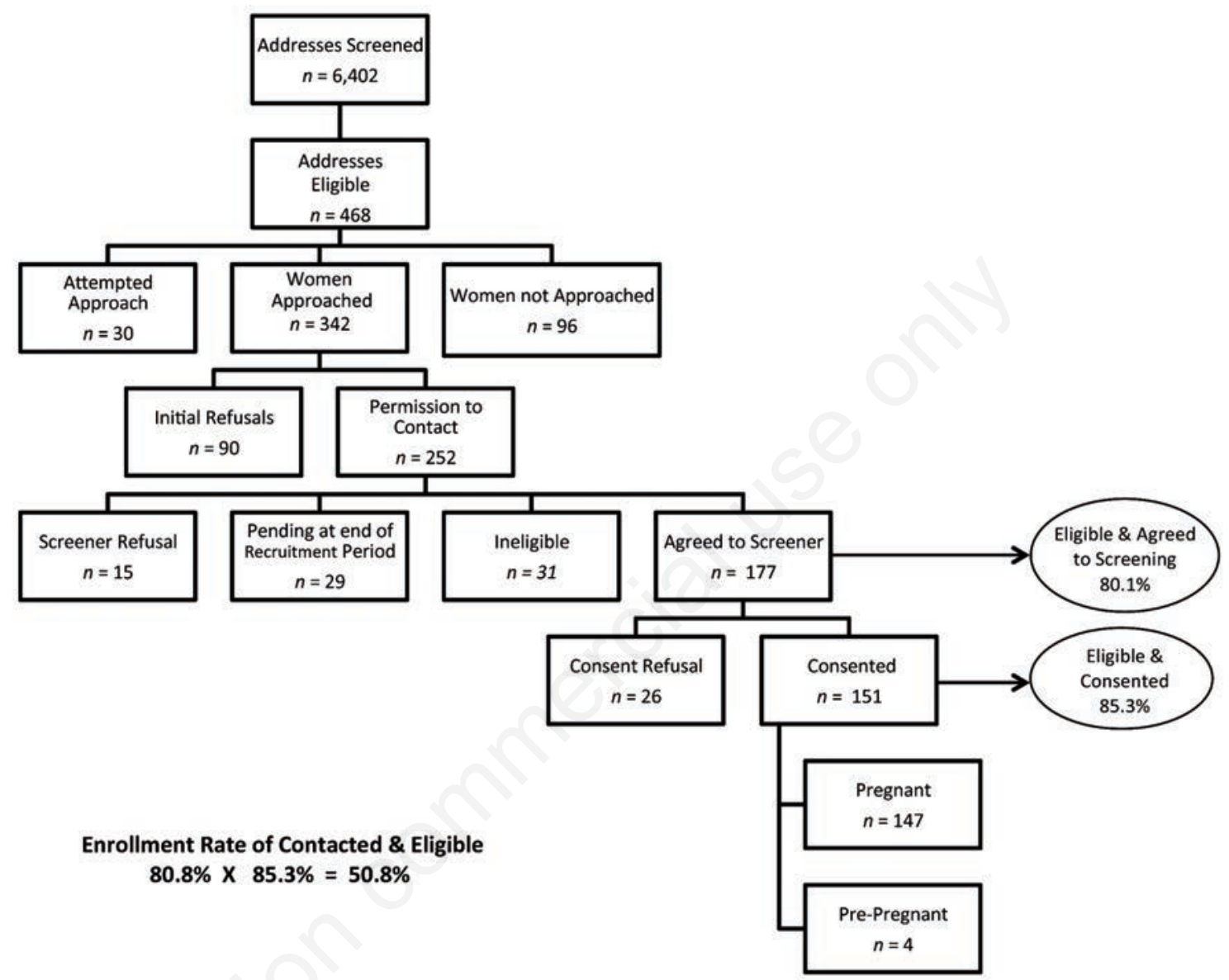

Figure 1. Benton County National Children's Study recruitment outcomes.

Table 1. Retention and event completion rates.

\begin{tabular}{lccc} 
& aRetention rate, $\%$ & bEvent completion rate, $\%$ & cEvent completion window not yet closed, $\%$ \\
Pregnancy visit 1 & 100 & 100 & - \\
Pregnancy visit 2 & 98.6 & 100 & 0 \\
\hline Birth & 97.3 & 99 & 0 \\
3 months & 97.3 & 99 & 0 \\
\hline 6 months & 96 & 97 & 1.4 \\
9 months & 96 & 98 & 12.7 \\
\hline 12 months & 96 & 98 & 44.3 \\
18 months & 95.2 & 100 & 23.6 \\
\hline 24 months & 95.2 & 100 &
\end{tabular}

aRate includes mothers of enrolled children ( $\mathrm{n}=147)$. bPercent of actively enrolled, event-eligible participants who completed the event. cStill event eligible at the end of data collection period March 2013. 
recruited from six clinics serving a mostly privately insured population and women recruited from the three clinics focusing on Medicaid or undocumented women. Women recruited from public clinics were significantly different than their private clinic counterparts with regard to race/ethnicity, education, age, marital status, and number of prenatal care visits. A higher percent of public clinic recruits were Hispanic, less educated, younger, unmarried, and had fewer prenatal visits. To determine whether pre-determined geographic segments produced more or less equal numbers of new births in each segment as intended by the sampling design, Arkansas birth records were analyzed for births per segment within the recruitment period. While 10 of the 14 segments had 13 to 23 births each (mean 18.1), two segments had 25 and 29 births, one segment had 9 births, and surprisingly one segment had 85 births.

Enrollment varied across segments. Because of phased clinic engagement over the 10 months of active recruitment, we did not expect to capture all or most of the births to segment residents. Of all segment-eligible births during the recruitment period $(n=329)$, $45 \%$ of births $(n=147)$ were successfully captured. Table 4 illustrates the distribution of eligible births, enrolled births and capture rate per segment. Between 30 and 50 percent of eligible women were enrolled from seven segments. Fewer than $20 \%$ of eligible women were enrolled from three segments, and between 53 and 85 percent of eligible women were enrolled from four segments.

Initial recruitment efforts at each newly engaged clinic yielded a large number of existent pregnant women, many of whom were in their second or third trimester. As recruitment proceeded, primarily newly pregnant women were identified and screened. Therefore, a bolus in gestational age at consent was expected and observed as clinics gradually came on line. This resulted in a significant decline in weeks of gestation of enrolled women over the engagement period from a mean of 25.5 weeks during the first 3 months of recruitment at a given clinic to a mean of 19.2 weeks in the remaining months.

\section{Discussion}

Employing a provider-based recruitment method based on pre-determined geographic eligibility, $68 \%$ of contacted eligible women were enrolled into a study to follow their new- born infants for 21 years of their lives. Given a growing reluctance of the population to participate in increasingly complex epidemiologic research and the required commitment to ongoing follow up for 21 years, 4,5 the participation of two-thirds of contacted eligible women is considerable. Recruitment rates of all eligible women from the seven original NCS vanguard centers (61\%) and one other NCS provider-based recruitment site (75\%) were similar to the rate observed here, ${ }^{6-9}$ and within the published range of recruitment (25-74\%) reported by other prospective pregnancy studies with similar recruitment methods. $5,10-20$

Women enrolled in the NCS from Benton County were similar to non-enrolled segment eligible women across most socio-demographic characteristics with the exception of fewer than expected Asian or Pacific Islander mothers and more than expected college graduates. Educational results are similar to the combined prenatal and preconception cohort recruited into the Right from the Start study and the prenatal cohort enrolled in the Pregnancy, Infection, and Nutrition study. ${ }^{15,16}$ In contrast to the Wayne County NCS sample that included significantly more black women, lower educated women, and women who received fewer prenatal visits than nonenrolled eligible births, ${ }^{8}$ the Benton County

Table 2. Socio-economic and demographic characteristics of Benton County participants compared to non-recruited segment-eligible mothers during the same period.

\begin{tabular}{|c|c|c|c|}
\hline Characteristics & Benton County participants ( $\mathrm{n}=147)$ & Non-recruited segment mothers $(\mathrm{n}=328)$ & RR $[95 \% \text { CI }]^{\mathrm{a}}$ \\
\hline \multicolumn{4}{|l|}{ Race/ethnicity, \% } \\
\hline White/Non-Hispanic & 70.3 & 70.4 & reference \\
\hline Black/Non-Hispanic & $*$ & 2.7 & $0.72[0.23,2.26]$ \\
\hline Asian or Pacific Islander & * & 8.8 & $0.38[0.15,0.95]$ \\
\hline Hispanic & 19.6 & 16.2 & $1.55[0.85,2.83]$ \\
\hline Other & $*$ & 1.8 & $1.46[0.33,6.49]$ \\
\hline \multicolumn{4}{|l|}{ Education, \% } \\
\hline Less than High-School & 12.2 & 11.4 & $1.41[0.68,2.91]$ \\
\hline High School & 24.5 & 38 & reference \\
\hline Some College & 13.6 & 19.1 & $1.26[0.65,2.46]$ \\
\hline College & 49.7 & 31.5 & $2.59[1.45,4.63]$ \\
\hline \multicolumn{4}{|l|}{ Age (years) } \\
\hline $18-24$ & 21.6 & 33.8 & reference \\
\hline $25-34$ & 66.9 & 55.8 & $1.30[0.75,2.26]$ \\
\hline $35+$ & 11.5 & 10.4 & $1.32[0.61,2.85]$ \\
\hline \multicolumn{4}{|l|}{ Marital status } \\
\hline Married & 80.4 & 75 & reference \\
\hline Unmarried & 19.6 & 25 & $1.07[0.60,1.92]$ \\
\hline \multicolumn{4}{|l|}{ Number of prenatal visits, $\%$} \\
\hline $10+$ & 88 & 80.9 & reference \\
\hline$<10$ & 12 & 19.1 & $0.61[0.33,1.13]$ \\
\hline Gestational age, \% mean (SD) & $38.7(1.8)$ & $38.3(2.1)$ & \\
\hline 37 + weeks & 92.6 & 91.4 & reference \\
\hline$<37$ weeks & 7.4 & 8.6 & $0.81[0.33,2.04]$ \\
\hline Birth weight, \% mean (SD) & $3.4(0.5)$ & $3.3(0.6)$ & \\
\hline $2.5+\mathrm{kg}$ & 94.7 & 92.8 & reference \\
\hline Less than $2.5 \mathrm{~kg}$ & * & 7.2 & $1.20[0.40,3.57]$ \\
\hline
\end{tabular}

aRisk ratios (RR) adjusted by multivariable binary regression with a log link for maternal age, race/ethnicity, education, marital status, number of prenatal visits, gestational age, and birth weight. ${ }^{*}$ Counts of $<10$ that can be derived from percentages are not presented in accordance with the NCS Data Access and Confidentiality policy. 
enrolled sample and non-enrolled births were similar across these characteristics. Underrepresentation of infants born to Asian and Pacific Islander mothers could bias the enrolled sample toward better perinatal outcomes. Previous research has shown these ethnic groups to have higher than normal risk of pre-term delivery, gestational diabetes, low birth weight, and macrosomia. ${ }^{21}$

Inclusion of the two federally qualified health centers and the county health department prenatal clinic in the provider mix effectively eliminated the potential bias of sampling pregnant women only from private clinics. Enrollment of women from these clinics resulted in a final sample closely approximating the birth population in race/ethnicity, age, marital status, number of prenatal visits, and gestational age and birth weight of their newborns. Promislow and colleagues have also shown that recruitment of pre-pregnant and pregnant women from both public and private prenatal clinics is essential for appropriate representation of minorities, lower income, and those with less favorable health behaviors. ${ }^{16}$

By design Benton County was partitioned into geographic segments of approximately equal numbers of births based on past birth records. Historical births were geocoded according to mother's place of residence to assign births to segments. Births from 2001 through 2006 were the most recent set of 6 years of data available to us at the time of the contract submission in 2008. Of 126 segments generated, subject eligibility was restricted to 14 randomly chosen segments. Unfortunately the geographic distribution of women of reproductive age in the county changed significantly by the time the study entered the field in 2011 . From 2001 to 2010 births to residents of Benton County grew from 2197 to 3234 . Perhaps because of new residential developments, one segment in particular was home to over four times the number of births in 2011 compared to the average across the remaining 13 sampled segments. Outdated information on the geographic distribution of births combined with new residential distribution led to unintended consequences of unequal geographic eligibility across the county. A similar outcome was observed in the Queens, New York NCS site. Use of birth records 5 to 7 years out of date to construct geographic segments resulted in capture rates that varied among segments from 8 to $96 \%{ }^{7}$

In the 10 months of active recruitment plus five months of passive recruitment over 150\% of the targeted number of women and infants were enrolled into the National Children's Study. Success of this recruitment experiment can be traced primarily to the characteristics of the county and early operations decisions. Among all 105 counties sampled for the National Children's Study, the annual birth population of 3234 in Benton County is smaller than 61. Only nine provider groups and 50 unique providers offer prenatal care to county women. Compared to counties with 25,000 or more births per year and as many as 600 providers, ${ }^{8}$ the scope of the operational chal-

lenges of Benton County were easily manageable. Early decisions by the Principal Investigator (CAH) and Project Director (PAM) to assemble a data collection team with sufficient representation by well-trained nurse professionals allowed for easier integration of the

Table 3. Socio-economic and demographic characteristics of Benton County participants by clinic type. Sample sizes are not presented to comply with NCS Data Access and Confidentiality policy.

\begin{tabular}{|c|c|c|c|}
\hline & & ints & P value \\
\hline & 3 public clinics* & 6 private clinics & \\
\hline Race/ethnicity, \% & & & $<0.01$ \\
\hline White/non-Hispanic & 33.3 & 75.4 & \\
\hline Black/non-Hispanic & 0 & 4 & \\
\hline Asian or Pacific Islander & 0 & 4.8 & \\
\hline Hispanic & 66.7 & 13.5 & \\
\hline Other & 0 & 2.4 & \\
\hline Education, \% & & & $<0.01$ \\
\hline Less than HS & 44.4 & 8 & \\
\hline High School & 50 & 20.8 & \\
\hline Some College & 0 & 14.4 & \\
\hline College & 5.6 & 56.8 & \\
\hline Age, $\%$ & & $<0.01$ & \\
\hline $18-24$ & 44.4 & 17.5 & \\
\hline $25-34$ & 33.3 & 72.2 & \\
\hline $35+$ & 22.2 & 10.3 & \\
\hline Marital status, $\%$ & & & $<0.01$ \\
\hline Married & 50 & 84.9 & \\
\hline Unmarried & 50 & 15.1 & \\
\hline Number of prenatal visits, $\%$ & & & $<0.05$ \\
\hline$<10$ & 29.4 & 9 & \\
\hline $10+$ & 70.6 & 91 & \\
\hline Gestational age, $\%$ mean (SD) & $38.5(1.2)$ & $38.7(1.9)$ & 0.69 \\
\hline$<37$ weeks & 5.9 & 7.9 & \\
\hline $37+$ weeks & 94.1 & 92.1 & \\
\hline Birth weight, \% mean (SD) & $3.5(0.6)$ & $3.4(0.5)$ & 0.96 \\
\hline$<2.5 \mathrm{~kg}$ & 5.6 & 5.5 & \\
\hline $2.5 \mathrm{~kg}+$ & 94.4 & 94.5 & \\
\hline
\end{tabular}

Table 4. Eligible births, enrolled births, and capture rate by segment. Mothers of twins were only counted in her segment once.

Bligible for recruitment Enrolled births Capture rate, \%

\begin{tabular}{lccc} 
Segment 1 & 23 & 4 & 17.4 \\
Segment 2 & 14 & 7 & 50.0 \\
\hline Segment 3 & 12 & 8 & 66.6 \\
Segment 4 & 22 & 4 & 18.2 \\
\hline Segment 5 & 9 & 4 & 44.4 \\
Segment 6 & 25 & 10 & 40.0 \\
\hline Segment 7 & 22 & 7 & 31.8 \\
Segment 8 & 23 & 11 & 47.8 \\
\hline Segment 9 & 13 & 11 & 84.6 \\
Segment 10 & 15 & 8 & 53.3 \\
\hline Segment 11 & 16 & 3 & 18.8 \\
Segment 12 & 85 & 52 & 61.1 \\
\hline Segment 13 & 21 & 6 & 28.6 \\
Segment 14 & 29 & 14 & 48.4 \\
\hline Total & 329 & 149 & 45.3
\end{tabular}


project into the routine patient flow of the clinic. The decision to pair research nurse interviewers with research associate interviewers and to assign each pair to a set of clinics and a set of research subjects served to assure the integrity and continuity of the study and the comfort and familiarity of research subjects with program staff. ${ }^{5}$ Similar to other studies of recruitment from prenatal care providers, ${ }^{17}$ an individualized approach to procedures within each clinic was a necessity. Weekly or biweekly meetings with clinic managers ensured best practice by trouble shooting processes as a team. While largely successful, the Benton County experience has also been met with a series of challenges, most attributable to a large multicenter study developing over time. First, the secondary sampling unit of geographic segments within the primary sampling unit of the county necessitated the investigation of over 6000 potential addresses to generate about 470 geographically eligible potential subjects. Secondary sampling in smaller geographic segments was likely a hold over from the household-based sampling schema employed by the seven original vanguard centers. Theoretically, door-to-door sampling from households could be made more efficient by clustering eligible households within blocks or neighborhoods. These efficiencies were not realized with provider-based recruitment, were likely not necessary, and did not produce the expected equal distribution of births across geographic areas. In larger counties, geographic sampling would likely entail great effort with little gain.

Second, multiple changes in data collection instruments and protocols occurred in the 10 months of subject recruitment. Each change required a new submission to the UAMS IRB 37 unique submissions in all. Each change also required numerous new programming tasks to update the computer assisted data collection instruments.

Third, operation of this study within the constraints of a federal contract also presented unique challenges. Because of the Federal Information Security Management Act (FISMA), UAMS was required to develop an extensive data security plan. This plan took 9 months and many person hours to finalize. The policy of facilitated decentralization adopted by the program office also required that each Study Center expend considerable resources to develop their own information management system.

Engagement of providers was extremely successful in Benton County. All agreed to participate. Fully $57 \%$ of counties initially sampled by NCS are of small to midsize similar to Benton, allowing for efficient engagement of all providers. In very large counties sampling from providers based on size of prenatal care practice could eliminate the necessity of a sec- ondary sampling unit. All patients from sampled providers who lived in the county would be eligible for inclusion. This potential design has been described by NCS Principal Investigators. 17,18

\section{Conclusions}

Enrolling pregnant women from prenatal care providers can be accomplished efficiently in geographic areas like Benton County where there are a manageable number of providers. Cooperation of providers and ultimately participants is highly dependent on qualified, welltrained staff that understands the operations of a busy clinic, can develop strong relationships with clinic staff, and can communicate the importance of the participation in a 21year study to potential subjects.

\section{References}

1. US Government. Public Law 106-310, October 17, 2000. H.R. 4365. Children's Health Act of 2000 .

2. Savitz DA, Zeger SL, Golding J, et al. Final report from the National Children's Study Sampling Design Workshop. Arlington, USA. May 9, 2004.

3. Guttmacher AE, Hirschfeld S, Collins FS. The National Children's Study. A proposed plan. New Engl J Med. 2013;369:1873-5.

4. Galea S, Tracy M. Participation rates in epidemiologic studies. Ann Epidemiol 2007;17:643-53.

5. Eskenazi B, Gladstone EA, Berkowitz GS, et al. Methodologic and logistic issues in conducting longitudinal birth cohort studies: lessons learned from the Centers for Children's Environmental Health and Disease Prevention Research. Environ Health Perspect 2005;113:1419-29.

6. Hirschfeld S, Songco D, Kramer BS, Guttmacher AE. National Children's Study: update in 2010. Mt Sinai J Med 2011;78:119-25.

7. Trasande L, Andrews HF, Goranson C, et al. Early experiences and predictors of recruitment success for the National Children's Study. Pediatrics 2011;127:2618.

8. Kerver JM, Elliott MR, Norman GS, et al. Pregnancy recruitment for population research: the National Children's Study vanguard experience in Wayne County, Michigan. Paediatr Perinat Epidemiol 2013;27:303-11.

9. Baker D, Park C, Sweeney C, et al. Recruitment of women in the National Children's Study initial vanguard study.
Am J Epidemiol 2014;179:1366-74.

10. Buck GM, Lynch CD, Stanford JB, et al. Prospective pregnancy study designs for assessing reproductive and developmental toxicants. Environ Health Perspect 2004;112:79-86

11. Buck Louis GM, Schisterman EF, Sweeney $\mathrm{AM}$, et al. Designing prospective cohort studies for assessing reproductive and developmental toxicity during sensitive windows of human reproduction and development - the LIFE Study. Paediatr Perinat Epidemiol 2011;25:413-24.

12. Eskenazi B, Bradman A, Gladstone EA, et al. CHAMACOS, a longitudinal birth cohort study: lessons from the fields. J Child Health 2003;1:3-27.

13. Golding J, Birmingham K. Enrollment and response rates in a longitudinal birth cohort. Paediatr Perinat Epidemiol 2009; 23:73-85.

14. Nohr EA, Frydenberg M, Henriksen TB, Olsen J. Does low participation in cohort studies induce bias? Epidemiology 2006;17:413-8.

15. Savitz DA, Dole N, Williams J, et al. Determinants of participation in an epidemiological study of preterm delivery. Paediatr Perinat Epidemiol 1999;13:11425.

16. Promislow JH, Makarushka CM, Gorman JR, et al. Recruitment for a communitybased study of early pregnancy: the right from the start study. Paediatr Perinat Epidemiol 2004;18:143-52.

17. Belanger K, Buka S, Cherry DC, et al. Implementing provider-based sampling for the National Children's Study: opportunities and challenges. Paediatr Perinat Epidemiol 2013;27:20-6.

18. Annett RD, Baker D, Bancroft J, et al. A cost-effective and feasible design for the National Children's Study (NCS): recommendations from the field. 2013. Available from: http:/blogs.nature.com/news/ files/ 2012/04/A-Cost-Effective-and-FeasibleDesign-for-the-National-Children\% E2\%80\%99s-Study-Recommendationsfrom-the-Field1.pdf

19. Webster GM, Teschke K, Janssen PA. Recruitment of healthy first-trimester pregnant women: lessons from the Chemicals, Health and Pregnancy Study (CHirP). Matern Child Health J 2012;16:430-8.

20. Handler A, Rosenberg D, Johnson T, et al. Prospective recruitment of women receiving prenatal care from diverse provider arrangements: a potential strategy. Matern Child Health J 1997;1:173-7.

21. Rao AK, Daniels K, El-Sayed YY, et al. Perinatal outcomes among Asian American and Pacific Islander women. Am J Obstet Gynecol 2006;195:834-8. 